ESAIM: PROCEEDINGS, May 2009, Vol. 27, p. 1-14

C. Besse, O. Goubet, T. Goudon \& S. Nicaise, Editors

\title{
ANISOTROPY AND VORTICES IN BOSE EINSTEIN CONDENSATES
}

\author{
Amandine Aftalion ${ }^{1}$
}

\begin{abstract}
The fast rotation of a Bose Einstein condensate in a harmonic trap leads to a hexagonal lattice of vortices, called the Abrikosov lattice. Vortices are described as the zeros of the condensate wave function, which is a solution to a nonlinear Schrdinger equation type. We study the solutions of this equation on a specific eigenspace of the limiting problem, the Lowest Landau Level. Upper and lower bounds for the energy allow to determine the presence of a vortex lattice or the lack of visible vortices, as a function of the anisotropy and the rotation. The study of the critical regime is open and would need some numerical simulations. This constitutes a project in collaboration with Xavier Blanc and Nicolas Lerner.
\end{abstract}

\section{IntRoduction}

Vortices in classical fluids are often related to turbulent phenomena, in hurricanes or typhoons for instance. In quantum fluids, such as Bose Einstein condensates (BEC), quantized vortices appear when the fluid is rotating and they provide an evidence of superfluidity. The aim of this paper is to make a review of mathematical issues related to vortices in fast rotating BEC. This is based on the papers $[4,6-8]$.

\subsection{Physical context}

The phenomenon of condensation was predicted in 1925 by Einstein, on the basis of a paper by Bose: for a gas of non interacting particles, below a certain temperature, there is a phase transition where a macroscopic fraction of the gas gets condensed, that is, all the atoms occupy the state of lowest energy and are described by the same wave function, which is also the wave function of the condensate. There was no experimental evidence of this phenomenon, since at the densities and temperature required by the theory, all materials were in the solid state. The development of cooling techniques [10] led in 1995, to the achievement of Bose Einstein condensation in atomic gases by the Jila group in Boulder (Cornell, Wiemann [12]), and very soon afterwards by the MIT group (Ketterle [15]), which was awarded with the Nobel Prize for Physics in 2001. Now, many properties of these systems are studied both experimentally and theoretically. We refer to the books by PethickSmith [19] and Pitaevskii-Stringari [20] for more details on the physics. An important issue is the relationship between Bose Einstein condensation and superfluidity, in particular through the existence of vortices. A BEC is a quantum macroscopic object described by a macroscopic wave function $\psi$ (complex-valued order parameter). For $\psi=\sqrt{\rho} e^{i S}$, vortices are lines of singularities along which $\rho=0$ and around which $(1 /(2 \pi)) \oint \nabla S \cdot d r$ is an integer: $\nabla S$ is a superfluid velocity. There are two classical experiments which illustrate the quantum fluid behaviour and are addressed on a mathematical ground in a book by the author [2]. One consists in stirring a laser beam along the condensate in a translation movement: there is a critical velocity below which the movement is dissipationless and beyond which the stirring produces vortices, as in the moving of an object

${ }^{1}$ CNRS, Université Pierre et Marie Curie-Paris6, UMR 7598, Laboratoire Jacques-Louis Lions, 175 rue du Chevaleret, Paris F-75013 France

(c) EDP Sciences, SMAI 2009 
in a superfluid. These aspects are described in [2], and will not be addressed here. In another experiment, achieved in the group of Jean Dalibard in Paris [17], a trapped system, which has a cigar shape, is rotated with a "spoon", like coffee in a cup. For small angular velocities, no modification of the condensate is observed. For sufficiently large angular velocities, the condensate behaves like a quantum fluid and, unlike coffee, vortices are detected in the system. When the rotational velocity is increased, the number of singularity lines increases and they get ordered through an Abrikosov lattice [1,9] (see Figure 1). The study of the lattice is at the core of very recent works $[5,11,13,14,23]$ in the condensed matter physics community. We intend to address these aspects mathematically here.

\subsection{Mathematical setting}

The study of interacting nonuniform quantum gases at zero temperature can be made in the framework of the Gross-Pitaevskii energy. The field operator used to describe quantum phenomena can be replaced by a classical field $\psi$, also called the order parameter or wave function of the condensate. We are interested in stationary phenomena: thus in the frame rotating at angular velocity $\tilde{\boldsymbol{\Omega}}=\tilde{\Omega} \mathbf{e}_{3}$, the trapping potential and the wave function are time independent. In the experimental device, the rotation is obtained thanks to a laser beam imposed on the magnetic trap holding the atoms to create a harmonic anisotropic rotating potential. The wave function $\psi$ minimizes the following energy, called the Gross Pitaevskii energy, which includes in this order a kinetic contribution, a term due to rotation, a term due to the presence of a harmonic trapping and a term due to atomic interactions:

$$
\int_{\mathbb{R}^{3}} \frac{\hbar^{2}}{2 m}|\nabla \psi|^{2}-\hbar \tilde{\mathbf{\Omega}} \times \mathbf{x} \cdot(i \psi, \nabla \psi)+\frac{m}{2} \sum_{i=j}^{3} \omega_{j}^{2} x_{j}^{2}|\psi|^{2}+N g_{3 D}|\psi|^{4}
$$

under the constraint $\int|\psi|^{2}=1$. Here, $g_{3 D}=4 \pi \hbar^{2} a_{0} / m$, where $a_{0}$ is the scattering length describing interactions, $N$ is the number of atoms, $m$ is the atomic mass, $\mathbf{x}=\left(x_{1}, x_{2}, x_{3}\right), \omega_{j}$ is the trapping frequency along the $j$ th direction, and $(i \psi, \nabla \psi)$ denotes the scalar product in $\mathbb{C}$ so that it is equal to $(i \psi \nabla \bar{\psi}-i \bar{\psi} \nabla \psi) / 2$.

We define $\omega=\sqrt{\left(\omega_{1}^{2}+\omega_{2}^{2}\right) / 2}, d=(\hbar / m \omega)^{1 / 2}$, the characteristic length of the harmonic oscillator. Rescaling distances by $d$, that is setting $\phi(\mathbf{x})=d^{3 / 2} \psi(d \mathbf{x})$, and $\Omega=\tilde{\Omega} / \omega_{1}$, the energy becomes, in units of $\hbar \omega$,

$$
E_{3 D}(\phi)=\int_{\mathbb{R}^{3}} \frac{1}{2}|\nabla \phi|^{2}-\boldsymbol{\Omega} \times \mathbf{x} \cdot(i \phi, \nabla \phi)+\frac{1}{2}\left(\left(1-\nu^{2}\right) x_{1}^{2}+\left(1+\nu^{2}\right) x_{2}^{2}+\beta^{2} x_{3}^{2}\right)|\phi|^{2}+4 \pi N a_{0}|\phi|^{4}
$$

under $\int|\phi|^{2}=1$. We have set $1-\nu^{2}=\omega_{1}^{2} / \omega^{2}$ and $\beta=\omega_{3} / \omega$. If $\Omega>\sqrt{1-\nu^{2}}$, the energy is not bounded below: the rotating force is stronger than the trapping potential. Our asymptotic regime of fast rotation will be when $\Omega$ reaches $\Omega_{c}=\sqrt{1-\nu^{2}}$.

There are two distinct regimes of rotation according to the value of $\Omega$. For low rotational velocity, there is a transition from none to a few vortices in the system, and their characteristic size is much smaller than their inter distance. We refer to [2] for more details on this regime also known as the Thomas Fermi regime, which requires the introduction of a small parameter. The other regime is at high rotational velocity, that is when $\Omega$ is close to the critical trapping frequency $\Omega_{c}=\sqrt{1-\nu^{2}}$. Then, the centrifugal force nearly balances the trapping force, the condensate expands and the number of vortices diverges. There is a dense lattice for which vortices have approximately the same size as their inter distance.

The issue is thus to minimize the energy $E_{3 D}$, under $L^{2}$ norm equal to 1 . We refer to [2] for some results that will not be described here: we have identified the critical velocity for the nucleations of vortices; in the case of a single vortex, we have proved that it is not straight along the axis of rotation, but bending; its shape is characterized by an equation that we obtain through $\Gamma$ convergence. In the case of fast rotation, we want to investigate the vortex lattice. The characterization of the lattice as a mathematical object requires the introduction of Hilbert spaces adapted to the problem, and in particular Bargmann spaces. In this paper, we will describe the case of isotropic traps $(\nu=0)$ and the effect of anisotropy. 


\subsection{Main results}

We set $\nu=0$ in the energy (2) and write the first two terms as the expansion of a complete square, and thus subtract the extra term to find:

$$
E_{3 D}(\psi)=\int_{\mathbb{R}^{3}} \frac{1}{2}|\nabla \psi-i \Omega \times \mathbf{x} \psi|^{2}+\frac{1}{2}\left(1-\Omega^{2}\right)|r|^{2}|\psi|^{2}+\frac{1}{2} \beta^{2} x_{3}^{2}|\psi|^{2}+4 \pi N a_{0}|\psi|^{4},
$$

where $r=\left(x_{1}, x_{2}\right)$, under the constraint $\int_{\mathbb{R}^{3}}|\psi|^{2}=1$. We assume that $\psi \in \mathcal{H}_{3 D}$ defined by

$$
\mathcal{H}_{3 D}=\left\{\psi \in H^{1}\left(\mathbb{R}^{3}\right) \text {, s.t. } E_{3 D}(\psi)<\infty\right\}
$$

Our regime of interest is when $\Omega$ tends to 1 . It displays a strong analogy with Quantum Hall physics: the first part of the Gross Pitaevskii energy is similar to that of a charged particle in a uniform magnetic field. Therefore, the ground state becomes degenerate, as in the case of the Landau levels obtained for the motion of a charge in a magnetic field.

We prove that any minimizer $\psi$ is close to a product $u\left(x_{1}, x_{2}\right) \xi\left(x_{3}\right)$, where $\xi$ is a gaussian and $u$ is in the Lowest Landau level, that is the space

$$
L=\left\{u\left(x_{1}, x_{2}\right) \in L^{2}\left(\mathbb{R}^{2}\right) \text { s.t. } u\left(x_{1}, x_{2}\right)=f(z) e^{-|z|^{2} / 2}, f \text { holomorphic, } z=x_{1}+i x_{2}\right\}
$$

This implies in particular that vortices are almost straight. We study the minimization of the energy restricted to the Lowest Landau level which simplifies to

$$
\int_{\mathbb{R}^{2}}(1-\Omega)|r|^{2}|u|^{2}+\frac{a}{2}|u|^{4}
$$

for some $a$. We construct an upper bound with a triangular vortex lattice, and an averaged behaviour on large circles which is like an inverted parabola. We perform this analysis with the introduction of Bargmann spaces.

Then we analyze the case when $\nu \neq 0$ : our mathematical analysis yields that an anisotropy of the trap can drastically change the picture in the fast rotation regime. In this case, the condensate becomes very elongated in one direction. Inspired by Fetter [13], we find that the description of the condensate can still be made in the framework of the lowest Landau level. This requires the diagonalization of the quadratic form in a simplectic basis. We show that the averaged behaviour on large circles and the distribution of the vortices are very different from the isotropic case: the behaviour is an inverted parabola in the deconfined direction, and a fixed Gaussian in the other direction. In particular, if the rotation is fast enough, there are no vortices in the central region of the condensate where the inverted parabola is supported. Contrary to the isotropic case, the wave function in the LLL can display an inverted parabola profile in one direction without visible vortices. This regime has not been reached experimentally yet and provides prospects for experiments. On the other hand, if the rotation is fast, but the anisotropy very small compared to the rotation, the behaviour is very similar to the isotropic case.

We are now going to describe briefly the most significant results.

\section{ISOTROPIC TRAPS, VORTEX LATTICE}

In the isotropic case, that is $\nu=0$, the fast rotation regime is when the rotational velocity $\Omega$ gets close to 1 . We minimize (3) under the constraint $\int_{\mathbb{R}^{3}}|\psi|^{2}=1$ for $\psi \in \mathcal{H}_{3 D}$ defined by (4). We are going to prove that we can reduce the minimization to a $2 \mathrm{D}$ problem, and a problem for functions in the Lowest Landau Level. In this framework, we will study the vortex lattice. 


\subsection{Dimensional reduction}

In the regime where $\Omega$ tends to 1 , we expect any minimizer $\psi$ of (3) under the constraint $\int_{\mathbb{R}^{3}}|\psi|^{2}=1$, to be close to a product $\phi\left(x_{1}, x_{2}\right) \xi\left(x_{3}\right)$, where $\xi$ is a gaussian corresponding the ground state of the energy in the third direction, $\xi\left(x_{3}\right)=(\beta /(2 \pi))^{1 / 4} e^{-\beta x_{3}^{2} / 2}$, and $\phi$ is almost a minimizer of the two dimensional problem corresponding to $(3)$, where the coefficient of the quartic term has been modified:

$$
E_{2 D}(\Phi)=\int_{\mathbb{R}^{2}} \frac{1}{2}\left|\nabla \Phi-i \Omega r^{\perp} \Phi\right|^{2}+\frac{1}{2}\left(1-\Omega^{2}\right)|r|^{2}|\Phi|^{2}+\frac{1}{2} a|\Phi|^{4} .
$$

We have set $r=\left(x_{1}, x_{2}\right), r^{\perp}=\left(-x_{2}, x_{1}\right)$, and $a=8 \pi N a_{0} \int_{\mathbb{R}} \xi^{4}$. The natural space of minimization for $E_{2 D}$ is

$$
\mathcal{H}_{2 D}=\left\{\Phi \in H^{1}\left(\mathbb{R}^{2}\right) \text {, s.t. } E_{2 D}(\Phi)<\infty\right\} .
$$

Recall that $E_{3 D}$ is given by (3). Let us define

$$
\begin{aligned}
& I_{3 D}(\Omega)=\inf \left\{E_{3 D}(\psi), \psi \in \mathcal{H}_{3 D}, \int_{\mathbb{R}^{3}}|\psi|^{2}=1\right\}, \\
& I_{2 D}(\Omega)=\inf \left\{E_{2 D}(\Phi), \Phi \in \mathcal{H}_{2 D}, \int_{\mathbb{R}^{2}}|\Phi|^{2}=1\right\} .
\end{aligned}
$$

In [4], we prove that $I_{3 D}$ is well described, as $\Omega$ tends to 1 , up to the second term in the expansion, by $I_{2 D}(\Omega)$, and $\beta$ the trapping frequency in the third direction:

Theorem 2.1. [4] For each $\Omega$, the minima $I_{3 D}(\Omega)$ and $I_{2 D}(\Omega)$ are achieved and, as $\Omega$ tends to 1 ,

$$
I_{3 D}(\Omega)-\left(\frac{\beta}{2}+I_{2 D}(\Omega)\right)=o(\sqrt{1-\Omega}) .
$$

Moreover, if $I_{3 D}(\Omega)$ is achieved for some $\psi$ then, there exists $\phi\left(x_{1}, x_{2}\right) \in \mathcal{H}_{2 D} \cap C^{0,1 / 2}\left(\mathbb{R}^{2}\right)$ such that $w=$ $\psi-\phi\left(x_{1}, x_{2}\right) \xi\left(x_{3}\right)$ has the property that $|w|$ tends to 0 in $H^{1}\left(\mathbb{R}^{3}\right) \cap C^{0,1 / 2}\left(\mathbb{R}^{3}\right)$ as $\Omega$ tends to 1 .

Let us comment on these results. The estimate (10) is meaningful because as we will see below,

$$
I_{2 D}(\Omega)-1=O(\sqrt{1-\Omega}) .
$$

This Theorem implies that the vortex lines of $\psi$ are almost straight and their location in a transverse plane is determined by the minimization of $E_{2 D}$ that we will address in the next section.

The proof is made by projecting $\psi$ onto the first eigenstate of $-\frac{d^{2}}{d t^{2}}+\beta^{2} t^{2}$ in $\mathbb{R}$ with $L^{2}$ norm equal to 1 . The corresponding eigenvalue is $\beta$ and the other eigenvalues are $(2 k+1) \beta, k \in \mathbf{N}$. Since the gap between two eigenvalues is much bigger than $E_{2 D}(\phi)-1$, we get that a minimizer of $E_{3 D}$ is confined on the lowest eigenstate of $-\frac{d^{2}}{d t^{2}}+\beta^{2} t^{2}$, that is $w$ is small in energy, and by elliptic estimates, in stronger norms.

This Theorem justifies the study of the minimum of $E_{2 D}$ in the next section. We know that $\phi$ obtained in the Theorem is almost minimizing but we would like to prove that it is close to the $2 D$ minimizer in some $C^{0}$ norm.

The aim is thus to study the minimization of $E_{2 D}$.

\subsection{Lowest Landau Level}

The first term in the energy (6) is identical to the energy of a particle placed in a uniform magnetic field $2 \Omega$. The minimizers for

$$
\int_{\mathbb{R}^{2}} \frac{1}{2}|\nabla \psi-i \boldsymbol{\Omega} \times \mathbf{r} \psi|^{2} \text { under } \int_{\mathbb{R}^{2}}|\psi|^{2}=1
$$


are well known through the study of the eigenvalues of the operator $-(\nabla-i \boldsymbol{\Omega} \times \mathbf{r})^{2}$. The minimum is $\Omega$ and the corresponding eigenspace is of infinite dimension and called the lowest Landau level (LLL). This can be studied using a change of gauge and a Fourier transform in one direction. The other eigenvalues are $(2 k+1) \Omega$, $k \in \mathbf{N}$. A basis of the first eigenspace is given by

$$
\psi\left(x_{1}, x_{2}\right)=P(z) e^{-\Omega|z|^{2} / 2} \text { with } z=x_{1}+i x_{2}
$$

where $P$ varies in a basis of polynomials. The closure of this space in $L^{2}\left(\mathbb{R}^{2}\right)$ is made up of functions of the type (13) where $P$ is a holomorphic function. In this framework, vortices are the zeroes of the polynomial or holomorphic function and are thus easy to identify.

We will see that as $\Omega$ approaches 1 , the second and third term in the energy (6) produce a contribution of order $\sqrt{1-\Omega}$, which is much smaller than the gap between two eigenvalues of $-(\nabla-i \Omega \times \mathbf{r})^{2}$, namely $2 \Omega$. This allows us to prove that we can restrict the minimization of the energy (6) to this eigenspace. In fact, we choose the space $L$ given by (5), which is independent of $\Omega$ and stable by the action of $-(\nabla-i \Omega \times \mathbf{r})^{2}+\left(1-\Omega^{2}\right) r^{2}$. If $v \in L$, then the energy (6) simplifies to

$$
E_{L L L}(v):=\Omega+\int_{\mathbb{R}^{2}}(1-\Omega)|r|^{2}|v|^{2}+\frac{a}{2}|v|^{4}
$$

Let us define

$$
\begin{aligned}
I_{L L L}(\Omega) & =\inf \left\{E_{L L L}(v), v \in L \cap \mathcal{H}_{2 D}, \int_{\mathbb{R}^{2}}|v|^{2}=1\right\} \\
& =\inf \left\{E_{2 D}(v), v \in L \cap \mathcal{H}_{2 D}, \int_{\mathbb{R}^{2}}|v|^{2}=1\right\}
\end{aligned}
$$

Theorem 2.2. [4] For each $\Omega$, the minimum $I_{L L L}(\Omega)$ is achieved and, as $\Omega$ tends to 1,

$$
I_{2 D}(\Omega)-I_{L L L}(\Omega)=o(\sqrt{1-\Omega})
$$

Moreover, if $I_{2 D}(\Omega)$ is achieved for some $\phi$ then, there exists $u\left(x_{1}, x_{2}\right)$ in $L$ such that $w=\phi-u$ is such that $|w|$ tends to 0 in $H^{1}\left(\mathbb{R}^{2}\right) \cap C^{0, \alpha}\left(\mathbb{R}^{2}\right)$, for all $\alpha \in(0,1)$, as $\Omega$ tends to 1 .

This estimate is meaningful up to second order in the expansion, since, as we will see below, as $\Omega$ tends to 1 ,

$$
I_{L L L}(\Omega)-1=O(\sqrt{1-\Omega}) .
$$

The space $L$ is related to the Fock Bargmann space $\mathcal{F}$, see [8]:

$$
\mathcal{F}=\left\{f \in L^{2}\left(\mathbf{C}, e^{-|z|^{2}} d z\right), \text { s.t } f \text { holomorphic }\right\} \text { with }\|f\|_{\mathcal{F}}^{2}=\int_{\mathbf{C}}|f(z)|^{2} e^{-|z|^{2}} d z
$$

where $d z$ denotes the Lebesgue measure $d x_{1} d x_{2}$. This is a Hilbert space endowed with the scalar product $\langle f, g\rangle_{\mathcal{F}}=\int_{\mathbb{R}^{2}} f(z) g(z) e^{-|z|^{2}}$. The projection of a general function $g(z, \bar{z})$ onto $\mathcal{F}$ is explicit. This provides the expression for the orthogonal projection from $L^{2}\left(\mathbb{R}^{2}\right)$ onto $L$, that we call $\Pi_{L}$ :

$$
\left[\Pi_{L} v\right](z)=\frac{e^{-|z|^{2} / 2}}{\pi} \int_{\mathbf{C}} e^{z \overline{z^{\prime}}} e^{-\left|z^{\prime}\right|^{2}} v\left(z^{\prime}, \overline{z^{\prime}}\right) d z^{\prime}
$$

for all $v \in L^{2}\left(\mathbb{R}^{2}\right)$, with $z=x_{1}+i x_{2}$ and $v(z, \bar{z})=v\left(x_{1}, x_{2}\right)$. In Theorem 2.2, $v$ is the projection of $\phi$ on $L$.

Our aim, in the next sections, is to derive qualitative properties of minimizers of (15). 

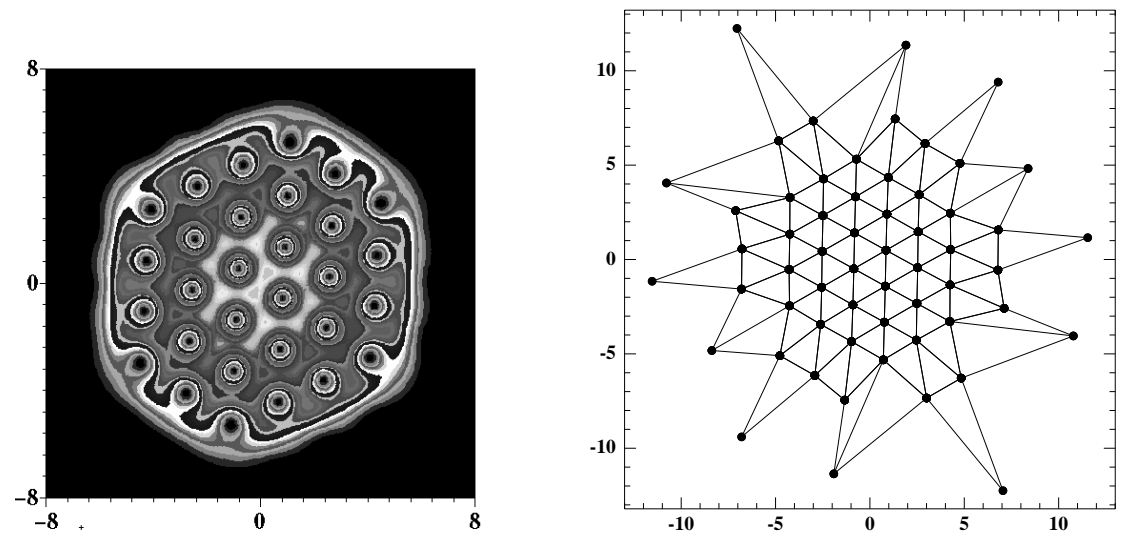

Figure 1. An example of (right): a configuration of $z_{i}$ minimizing the energy (14) for $\Omega=$ 0.999, $a=3$ and $n=58$. (left): density plot of $|v|$

\subsection{Shape of minimizers and main results}

We want to minimize the energy (14) under $\int_{\mathbb{R}^{2}}|v|^{2}=1$, when $v \in L$, that is study (15). A numerical study [5] consists in writing $v(z)=p_{0} \prod_{i=1}^{n}\left(z-z_{i}\right) e^{-|z|^{2} / 2}$ and minimize the energy on the location $z_{i}$ of the zeroes and the degree $n$. Our numerical observations indicate that vortices are located on a regular triangular lattice in a central region, while the lattice is distorted towards the edges, as illustrated in Figure 1 . On the right, we have plotted the $z_{i}$ and on the left $|v|$. The density plot of $|v|$ shows that the only visible vortices are the central ones in the regular lattice part, the outer ones being in a region of very low density. Our aim is to justify these observations rigorously. In $[3,5]$, we have constructed an upper bound for the energy inspired by our numerical computations.

\subsubsection{Inverted parabola profile}

Let us explain the origin of the inverted parabola profile: the minimization of (14) under $\int_{\mathbb{R}^{2}}|v|^{2}=1$ without the constraint of being in the space (5) implies that the minimizer is the inverted parabola

$$
\left|v_{\min }(r)\right|^{2}=\frac{2}{\pi R_{0}^{2}}\left(1-\frac{r^{2}}{R_{0}^{2}}\right) 1_{\left\{r \leq R_{0}\right\}}, \quad R_{0}=\left(\frac{2 a}{\pi(1-\Omega)}\right)^{1 / 4}
$$

The value of the energy yields a lower bound for $E_{L L L}$ :

$$
\epsilon_{\min }:=E_{L L L}\left(v_{\min }\right)-\Omega=\frac{2 \sqrt{2}}{3} \sqrt{a(1-\Omega)} .
$$

The function $v_{\min }$ does not belong to the space (5), since $f=v_{\min } e^{|z|^{2} / 2}$ is not holomorphic. Nevertheless, a distortion of the vortex lattice inside the space (5) provides a weak star approximation of the inverted parabola $v_{\text {min }}$ as $\Omega$ tends to 1 , but is going to modify the radius $R_{0}$ by a coefficient $b^{1 / 4}$ coming from the contribution of the lattice to the energy. Modifying the location of the vortices from a regular lattice can thus change the decay of the wave function and hence improve the energy estimate. This is what we want to understand. 


\subsubsection{Qualitative properties of minimizers}

We are going to rescale the problem. We let $R^{4}=a /(1-\Omega), h=1 / R^{2}$, and $u(\mathbf{r})=R v(R \mathbf{r})$. Then $E_{L L L}(v)=\Omega+\sqrt{a(1-\Omega)} E_{L L L}^{h}(u)$ where

$$
E_{L L L}^{h}(u)=\int_{\mathbf{C}}|z|^{2}|u|^{2}+\frac{1}{2}|u|^{4} d z
$$

where $d z$ denotes the Lebesgue measure $d x_{1} d x_{2}$. We want to minimize $E_{L L L}^{h}$ under the condition that $f(z)=$ $u(z) e^{\frac{|z|^{2}}{2 h}}$ is in the Fock Bargmann space $\mathcal{F}_{h}$, see [8]:

$$
\mathcal{F}_{h}=\left\{f \in L^{2}\left(\mathbf{C}, e^{-\frac{|z|^{2}}{2 h}} d z\right), \text { s.t } f \text { holomorphic }\right\} .
$$

The projection of a general function $g(z, \bar{z})$ onto $\mathcal{F}_{h}$ is explicit :

$$
\Pi_{h}(g)=\frac{1}{\pi h} \int_{\mathbb{R}^{2}} e^{\frac{z \bar{z}^{\prime}}{h}} e^{-\frac{\left|z^{\prime}\right|^{2}}{h}} g\left(z^{\prime}, \overline{z^{\prime}}\right) .
$$

If $g$ is a holomorphic function, then an integration by parts indeed yields $\Pi_{h}(g)=g$.

Using the properties of this space, we are able to derive an equation for the minimizer:

Theorem 2.3. [8] There exists $f \in \mathcal{F}_{h}$ such that $u(z)=f(z) e^{-|z|^{2} / 2 h}$ minimizes (22) with $\|f\|_{\mathcal{F}_{h}}=1$. Moreover, $f$ is a solution of the following equation

$$
\Pi_{h}\left(\left(|z|^{2}+|f|^{2} e^{-|z|^{2} / h}-\mu\right) f\right)=0
$$

where $\mu$ is the Lagrange multiplier coming from the constraint.

The Euler Lagrange equation can be rewritten

$$
z h \partial_{z} f+\frac{1}{2} \bar{f}\left(h \partial_{z}\right)\left[f^{2}\left(2^{-1} \cdot\right)\right]-(\mu-h) f=0,
$$

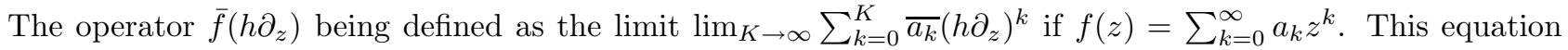
allows us to derive that $f$ cannot be a polynomial:

Theorem 2.4. [8] If $f \in \mathcal{F}_{h}$ is such that $u(z)=f(z / \sqrt{h}) e^{-|z|^{2} / 2}$ minimizes (15), then $f$ has an infinite number of zeroes.

The proof assumes that $f$ has a finite number of zeroes and derives a contradiction between the degree of the terms in (26).

We would like to prove that the zeroes of the minimizer are arranged on an almost regular lattice. For this purpose, we have to introduce the lattice problem.

\subsubsection{The Abrikosov problem}

We define a lattice $\mathfrak{L}$ in the following way:

$$
\mathfrak{L}=\frac{1}{\nu}(\mathbf{Z} \oplus \tau \mathbf{Z}), \quad \nu \in \mathbf{R}_{+}^{*}, \tau=\tau_{R}+i \tau_{I} \in \mathbf{C} .
$$

The functions $u$ such that $f(z)=u(z) e^{|z|^{2} / 2 h}$ is holomorphic, $|u|$ is periodic over the lattice $\mathfrak{L}$ and $u$ vanishes exactly on $\mathfrak{L}$ with simple zeroes, form a one dimensional space spanned by a function called $u_{\tau}$. This function is 
completely determined by the complex number $\tau$ of the lattice: indeed, the periodicity of $|u|$ imposes the value of $\nu$ in terms of $h$ and $\tau_{I}$. The function $u_{\tau}$ can be expressed in terms of the Theta function according to:

$$
\Theta(v, \tau)=\frac{1}{i} \sum_{n=-\infty}^{+\infty}(-1)^{n} e^{i \pi \tau(n+1 / 2)^{2}} e^{(2 n+1) \pi i v}, \quad v \in \mathbf{C} .
$$

Such an ansatz was introduced by Abrikosov to minimize the quantity

$$
\gamma(\tau)=\frac{f\left|u_{\tau}\right|^{4}}{\left(f\left|u_{\tau}\right|^{2}\right)^{2}}
$$

which also arises in the study of superconductors. It turns out that the square $(\tau=i)$ and hexagonal lattices $\left(\tau=e^{2 i \pi / 3}\right)$ are critical points of the function $\tau \rightarrow \gamma(\tau)$. The fact that the hexagonal lattice is a minimizer was numerically checked by Kleiner, Roth and Autler in [16]. An explicit computation of the quantity $\gamma(\tau)$, with the help of a result by Nonnenmacher and Voros [18] about quantum chaos, provides a complete proof that $\tau=e^{2 i \pi / 3}$ is the global minimizer:

Theorem 2.5. [8] Let $\mathfrak{L}$ be a lattice given by its parameters $\nu$ and $\tau$ through (27). If the function $f$ is entire, satisfies $f^{-1}(\{0\})=\mathfrak{L}$ with simple zeroes, and $\left|e^{-\frac{|z|^{2}}{2 h}} f(z)\right|$ is $\mathfrak{L}$-periodic, then the parameter $\nu$ and the function $f$ are determined by $\tau$ through:

$$
\begin{gathered}
\nu=\sqrt{\frac{\tau_{I}}{\pi h}} \text { and } f(z)=c f_{\tau}(z), \quad c \in \mathbf{C}^{*} \\
\text { with } \quad f_{\tau}(z)=e^{\frac{z^{2}}{2 h}} \Theta\left(\frac{\sqrt{\tau_{I}}}{\sqrt{\pi h}} z, \tau\right) \text { and } u_{\tau}(z)=e^{-\frac{|z|^{2}}{2 h}} f_{\tau}(z) .
\end{gathered}
$$

The function $f_{\tau}(z)$ solves the equation

$$
\begin{aligned}
& \Pi_{h}\left(e^{-\frac{|z|^{2}}{h}}\left|f_{\tau}\right|^{2} f_{\tau}\right)=\lambda_{\tau} f_{\tau}, \quad \text { in } \mathcal{F}_{h}^{s}, s<-1, \\
\text { with } \quad & \lambda_{\tau}=\frac{f\left|u_{\tau}\right|^{4}}{f\left|u_{\tau}\right|^{2}}=\frac{1}{\sqrt{2 \tau_{I}}} \sum_{k, \ell \in \mathbf{Z}} e^{-\frac{\pi}{\tau_{I}}|k \tau-\ell|^{2}}
\end{aligned}
$$

Moreover, the quantity $\gamma(\tau)$ defined in (28) satisfies

$$
\gamma(\tau)=\sum_{k, \ell \in \mathbf{Z}} e^{-\frac{\pi}{\tau_{I}}|k \tau-\ell|^{2}}
$$

The complex number $\tau=j=e^{\frac{2 i \pi}{3}}$, corresponding to the hexagonal lattice, is the unique minimizer of $\gamma(\tau)$ in the fundamental domain

$$
\left\{\tau=\tau_{R}+i \tau_{I} \in \mathbf{C}, \tau_{I}>0,|\tau| \geq 1,-\frac{1}{2} \leq \tau_{R}<\frac{1}{2},\left(\tau_{R} \leq 0 \text { if }|\tau|=1\right)\right\}
$$

and $b=\gamma(j) \sim 1.1596$

Let us point out that (30) is similar to (25) without the confining term. We expect that, when $h$ is sufficiently small, any minimizer of $(22)$ is close in some sense to $f_{\tau}(z) \alpha(z)$, where $f_{\tau}$ is the function described in Theorem 2.5 which varies on a characteristic size $\sqrt{h}$, and $\alpha$ is a slow varying profile which optimizes the energy and takes into account the confining potential. 


\subsubsection{Upper bound for the energy}

We prove that $f_{\tau}(z) \alpha(z)$ can be approximated, as $h$ tends to 0 , by the function $\Pi_{h}\left(\alpha f_{\tau}\right)$ of $\mathcal{F}_{h}$, which is almost a solution to $(25)$ :

Theorem 2.6. [8] Let $\tau \in \mathbf{C} \backslash \mathbf{R}$, let $\tilde{\alpha} \in C^{0, \frac{1}{2}}(\mathbf{C} ; \mathbf{C})$ be such that $\operatorname{supp}(\tilde{\alpha}) \subset K$ for some compact set $K$ and $\int|\tilde{\alpha}|^{2}=1$. For $f_{\tau}$ defined by (29), we set

$$
g_{\tilde{\alpha}, \tau}^{h}=\left\|\Pi_{h}\left(\tilde{\alpha} f_{\tau}\right)\right\|_{\mathcal{F}_{h}}^{-1} \Pi_{h}\left(\tilde{\alpha} f_{\tau}\right), \quad \text { and } \quad v_{\tilde{\alpha}, \tau}^{h}(z)=g_{\tilde{\alpha}, \tau}^{h}(z) e^{-\frac{|z|^{2}}{2 h}}
$$

Then we have

$$
E_{\mathrm{LLL}}^{h}\left(v_{\tilde{\alpha}, \tau}^{h}\right)=\int_{\mathbf{C}}\left(|z|^{2}|\tilde{\alpha}(z)|^{2}+\frac{\gamma(\tau)}{2}|\tilde{\alpha}(z)|^{4}\right) d z+O\left(h^{1 / 4}\right)
$$

where $\gamma(\tau)$ is given by (28) or (32) and $O\left(h^{1 / 4}\right)$ depends only on $\|\tilde{\alpha}\|_{C^{0,1 / 2}}, \tau$ and $K$. Moreover, for any $\lambda \in \mathbf{C}$,

$$
\Pi_{h}\left(\left(|z|^{2}-\lambda+\Omega_{h}^{2}\left|v_{\tilde{\alpha}, \tau}^{h}\right|^{2}\right) g_{\tilde{\alpha}, \tau}^{h}\right)=\Pi_{h}\left(\left(|z|^{2}-\lambda+\gamma(\tau)|\tilde{\alpha}|^{2}\right) g_{\tilde{\alpha}, \tau}^{h}\right)+R_{h}
$$

where $\left\|R_{h}\right\|_{\mathcal{F}_{h}} \leq C(\tilde{\alpha}, \tau, \lambda, K) h^{1 / 4}$, and $C(\tilde{\alpha}, \tau, \lambda, K)$ depends only on $\|\tilde{\alpha}\|_{C^{0,1 / 2}}, \tau, \lambda$ and $K$, and

$$
\left\|v_{\tilde{\alpha}, \tau}^{h}-\tilde{\alpha} u_{\tau}\right\|_{L^{\infty}(K)} \leq C h^{1 / 4}
$$

Let us point out that (35) shows that the projection of the nonlinear term provides the product of the average of $\left|u_{\tau}\right|^{4}$, hence $\gamma(\tau)$ times $|\tilde{\alpha}|^{2}$. The difference in the length scales $\left(\left|u_{\tau}\right|\right.$ varies on a scale $\sqrt{h}$ while $\tilde{\alpha}$ varies on a scale 1$)$ is taken into account to separate the contributions in the product.

In order to approximate a minimizer of $(22)$, we need to pick the optimal function $\tilde{\alpha}$. Minimizing the right-hand side of (34) with respect to $\tau$ and $\tilde{\alpha}$ under the constraint $\int|\tilde{\alpha}|^{2}=1$ yields

$$
\tau=j \text { and }|\tilde{\alpha}(z)|^{2}=\frac{1}{\gamma(\tau)}\left(\sqrt{\frac{2 \gamma(\tau)}{\pi}}-|z|^{2}\right)_{+}
$$

where the first equality is a consequence of Theorem 2.5. This provides in particular a test function for the upper bound of the energy (22), and in particular,

$$
I_{L L L}(\Omega)-\Omega \leq \frac{2 \sqrt{2}}{3} \sqrt{a b(1-\Omega)}
$$

and (34) makes precise the remainder estimate in the upper bound.

With this choice of $\tilde{\alpha}$ and $\tau$, and if in addition $\lambda$ in (35) is such that $\lambda=\sqrt{2 \gamma(\tau) / \pi},(35)$ implies that

$$
\Pi_{h}\left(\left(|z|^{2}-\lambda+\Omega_{h}^{2}\left|v_{\tilde{\alpha}, \tau}^{h}\right|^{2}\right) g_{\tilde{\alpha}, \tau}^{h}\right)=O\left(h^{1 / 4}\right) \quad \text { in } \quad \mathcal{F}_{h}
$$

In other words, $g_{\tilde{\alpha}, \tau}^{h}$ is a solution to $(25)$ up to an error term of order $h^{1 / 4}$. Moreover, as $h$ tends to $0, g_{\tilde{\alpha}, \tau}^{h}$ is very close to $f_{\tau}(z) \tilde{\alpha}(z)$. This implies that, inside the support of $\tilde{\alpha}$, the zeroes of $g_{\tilde{\alpha}, \tau}^{h}$ are located on an almost regular triangular lattice. We do not have much information though, on the zeroes located outside the support of $\tilde{\alpha}$, the "invisible vortices". An open question is to derive that there is a solution to $(25)$ close to $g_{\tilde{\alpha}, \tau}^{h}$, for the specific choice of $\tau$ and $\tilde{\alpha}$ given by (36). One may hope to prove such a result by an analogue of a Newton method.

Our results deal with an upper bound for the energy. A natural question would be to get also the lower bound and prove $\Gamma$ convergence type results. Recall that the lower bound (21) does not contain the coefficient 
$b=\gamma(j)$. We expect that reproducing an inverted parabola profile in the space (5) requires a lot of vortices and thus creates a contribution in the energy through $b$. But proving that the zeroes of the minimizer (we know by Theorem 2.4 that there is an infinite number) are located on an almost regular lattice seems very difficult and is probably related to similar difficulties in crystallization and sphere packing problems.

\section{Anisotropic CASE}

We go back to the energy (2) and assume that $\nu \neq 0$. We address the two-dimensional case only, that is do not enter into the details of the dimensional reduction leading to (6). The energy that we want to minimize is thus

$$
E_{a n}(\psi)=\int_{\mathbb{R}^{2}} \frac{1}{2}|\nabla \psi|^{2}-\boldsymbol{\Omega} \times \mathbf{x} \cdot(i \psi, \nabla \psi)+\frac{1}{2}\left(\left(1-\nu^{2}\right) x_{1}^{2}+\left(1+\nu^{2}\right) x_{2}^{2}\right)|\psi|^{2}+\frac{1}{2} G|\psi|^{4}
$$

under the $L^{2}$ constraint, with $G$ equal to $4 \pi N a_{0}$ times the $L^{4}$ norm of the ground state in the $z$ direction. The critical frequency provides the existence of a small parameter $\varepsilon$, with

$$
\varepsilon^{2}=1-\nu^{2}-\Omega^{2}
$$

We will see that an anisotropy of the trap can drastically change the picture in the fast rotation regime, that is small $\varepsilon$.

We would like to perform the same steps as in the isotropic case, that is find new coordinates (equivalent of the LLL) where the energy gets simplified and then study this reduced energy in order to understand the vortex behaviour. This is performed in $[6,7]$ and uses the diagonalization of the quadratic form in a simplectic basis, as we will see below, to find the reduced energy

$$
\mathcal{E}_{L L L}(\psi)=\int_{\mathbb{R}^{2}} \frac{1}{2}\left(\varepsilon^{2} x_{1}^{2}+\kappa^{2} x_{2}^{2}\right)|\psi|^{2}+\frac{G}{2}|\psi|^{4}
$$

with $\kappa^{2} \sim \frac{\left(\nu^{2}+\varepsilon^{2} / 2\right)\left(2-\nu^{2}\right)}{\left(1-\nu^{2}\right)}$ as $\varepsilon$ tends to $0 . \mathcal{E}_{L L L}$ is minimized on the space $L$ given by $(5)$.

Thus, we are interested in the following problem

$$
I(\varepsilon, \kappa)=\inf \left\{\mathcal{E}_{L L L}(\psi), \quad \psi \in L, \quad \int_{\mathbb{R}^{2}}|\psi|^{2}=1\right\} .
$$

As $\varepsilon$ tends to 0 , the condensate expands in the $x_{1}$ direction. In the $x_{2}$ direction, according to the values of $\varepsilon$ and $\nu$, either the condensate expands (inverted parabola profile) or stays bounded. Indeed, the minimization without the holomorphy constraint yields

$$
|\psi|^{2}=\frac{2}{\pi R_{1} R_{2}}\left(1-\frac{x_{1}^{2}}{R_{1}^{2}}-\frac{x_{2}^{2}}{R_{2}^{2}}\right), \text { where } R_{1}=\left(\frac{2 G \kappa}{\pi \varepsilon^{3}}\right)^{1 / 4}, \quad R_{2}=\left(\frac{2 G \varepsilon}{\pi \kappa^{3}}\right)^{1 / 4}
$$

There are 2 regimes:

- $\varepsilon^{1 / 3}>>\kappa: R_{2} \rightarrow \infty$. Numerical simulations (figure 2) show a triangular vortex lattice with an inverted parabola profile. The behaviour is similar to the isotropic case except that the inverted parabola $\alpha$ takes into account the anisotropy, $f=\Pi\left(\alpha(z) f_{\tau}(z)\right)$ is an approximate solution of the equation.

- $\varepsilon^{1 / 3}<<\kappa: R_{2} \rightarrow 0$. Numerical simulations (figure 3) show that the behaviour is an inverted parabola in the $x_{1}$ direction and a fixed gaussian in the $x_{2}$ direction. The shrinking of the condensate in the $x_{2}$ direction is not allowed in the lowest Landau level because the operator $x_{2}^{2}$ is bounded from below and the first eigenfunction is a gaussian in the $x_{2}$ direction. There are no vortices. We find an asymptotic 1D problem (upper and lower bound match) which yield a separation of variables. 

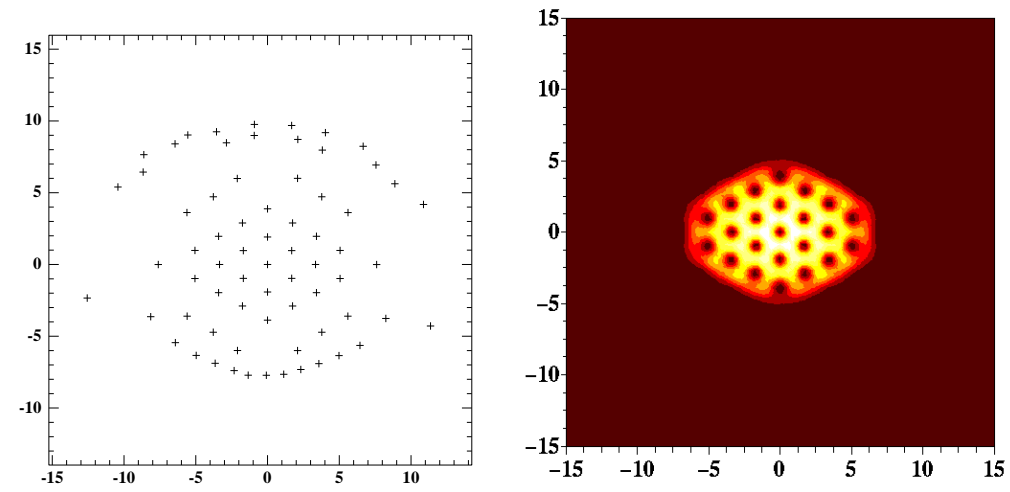

Figure 2. Plot of the zeroes of the minimizer (left) and the density (right) for $\varepsilon^{2}=0.002$, $\nu=0.0316$. Triangular vortex lattice in an anisotropic trap.
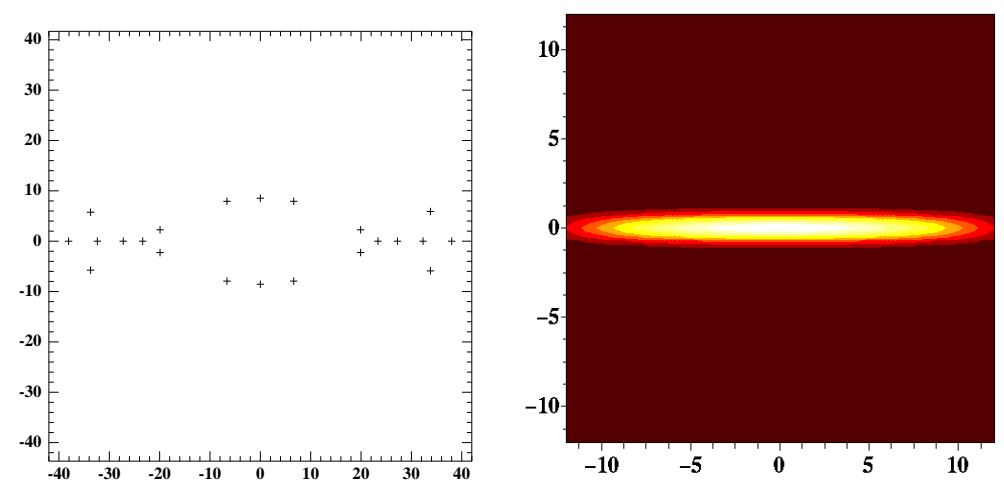

Figure 3. Plot of the zeroes of the minimizer (left) and the density (right) for $\varepsilon^{2}=0.002$, $\nu=0.06$. No vortex in the visible region.

\subsection{Reduction to an LLL energy}

In this section, we sketch the computations which allow to reduce $E_{a n}$ to $\mathcal{E}_{L L L}$ and the space $L$. We define the quadratic form corresponding to the linear terms in the energy:

$$
Q\left(x_{1}, x_{2}, \xi_{1}, \xi_{2}\right)=\xi_{1}^{2}+\xi_{2}^{2}+\left(1-\nu^{2}\right) x_{1}^{2}+\left(1+\nu^{2}\right) x_{2}^{2}-2 \Omega\left(x_{1} \xi_{2}-x_{2} \xi_{1}\right)
$$

We consider the phase space $\mathbb{R}_{x}^{2} \times \mathbb{R}_{\xi}^{2}$ with the symplectic form $\sigma$, which is a bilinear alternate form given by

$$
\sigma((x, \xi) ;(y, \eta))=\xi \cdot y-\eta \cdot x=<\sigma X Y>, X=\left(\begin{array}{l}
x \\
\xi
\end{array}\right), Y=\left(\begin{array}{l}
y \\
\eta
\end{array}\right), \sigma=\left(\begin{array}{cc}
0 & I_{2} \\
-I_{2} & 0
\end{array}\right)
$$

where the form $\sigma$ is identified with the $4 \times 4$ matrix above.

Given the quadratic form $\mathrm{Q}$, we define its fundamental matrix by the identity $F=-\sigma^{-1} Q=\sigma Q$. The properties of the eigenvalues and eigenvectors of $F$ allow to simplify $Q$ : the eigenvalues of $F$ are $\pm i \mu_{1}, \pm i \mu_{2}$, 
where

$$
\mu_{1}^{2}=1+\Omega^{2}-\sqrt{\nu^{4}+4 \Omega^{2}}, \quad \mu_{2}^{2}=1+\Omega^{2}+\sqrt{\nu^{4}+4 \Omega^{2}} .
$$

We define $\alpha=\sqrt{\nu^{4}+4 \Omega^{2}}, \beta_{1}=\left(2 \Omega \mu_{1}\right) /\left(\alpha-2 \Omega^{2}+\nu^{2}\right), \beta_{2}=\left(2 \Omega \mu_{2}\right) /\left(\alpha+2 \Omega^{2}+\nu^{2}\right), \gamma=(2 \alpha) / \Omega, \lambda_{1}^{2}=$ $\left(\alpha-2 \Omega^{2}+\nu^{2}\right) /(2 \alpha), \lambda_{2}^{2}=\left(\alpha+2 \Omega^{2}+\nu^{2}\right) / 2 \alpha, d=\left(\gamma \lambda_{1} \lambda_{2}\right) / 2, c=\left(\lambda_{1}^{2}+\lambda_{2}^{2}\right) / 2 \lambda_{1} \lambda_{2}$. Then computations lead to the following diagonalization of $Q$ :

$$
Q=\frac{1}{2}\left(a_{1}^{\dagger} a_{1}+a_{1} a_{1}^{\dagger}\right)+\frac{1}{2}\left(a_{2}^{\dagger} a_{2}+a_{2} a_{2}^{\dagger}\right)
$$

where

and

$$
a_{2}=\frac{\mu_{2}}{\sqrt{2}}\left(-i \lambda_{1} d^{-1} \partial_{x_{1}}+c \lambda_{1} x_{2}\right)+\frac{i}{\sqrt{2}}\left(-i \lambda_{2} \partial_{x_{2}}-\left(d \lambda_{1}^{-1}-\lambda_{2} c d\right) x_{1}\right)
$$

$$
a_{1}=\frac{\mu_{1}}{\sqrt{2}}\left(-i \lambda_{2} d^{-1} \partial_{x_{2}}+c \lambda_{2} x_{1}\right)+\frac{i}{\sqrt{2}}\left(\left(\lambda_{1} c d-d \lambda_{2}^{-1}\right) x_{2}-i \lambda_{1} \partial_{x_{1}}\right)
$$

We have: $\left[a_{2}, a_{2}^{\dagger}\right]=\mu_{2}, \quad\left[a_{1}, a_{1}^{\dagger}\right]=\mu_{1}$, and all other commutators vanish. Since $\mu_{1}<\mu_{2}$, the LLL is then defined by $a_{2} \psi=0$, that is $f\left(x+i \beta_{2} y\right) e^{\left[-\frac{1}{8 \beta_{2}}\left(\frac{2 \alpha-\nu^{2}}{\Omega} x^{2}+\frac{2 \alpha+\nu^{2}}{\Omega}\left(\beta_{2} y\right)^{2}\right)\right]-i \frac{\nu^{2}}{4 \Omega} x y}$, with $f$ analytic. In the LLL, we have $\langle Q \psi, \psi\rangle=\frac{1}{2}<\left(a_{1}^{\dagger} a_{1}+a_{1} a_{1}^{\dagger} \psi, \psi>+\frac{\mu_{2}}{2}<\psi, \psi>\cdot a_{1}, a_{2}, a_{1}^{\dagger}, a_{2}^{\dagger}\right.$ [13] and get, if $\psi \in L L L$,

$$
<Q \psi, \psi>=\frac{\mu_{2}}{2}-\frac{\mu_{1}}{4}\left(\beta_{1} \beta_{2}+\frac{1}{\beta_{1} \beta_{2}}\right)+\frac{\gamma}{4} \int_{\mathbb{R}^{2}}\left(\mu_{1} \beta_{1} x_{1}^{2}+\frac{\mu_{1}}{\beta_{1}} x_{2}^{2}\right)|\psi|^{2} d x_{1} d x_{2} .
$$

It is always possible to change the analytic function $f(\xi)$ into $f(\xi) \exp \left(-\delta \xi^{2}\right)$ in the above definition of the LLL, since $\exp \left(-\delta \xi^{2}\right)$ is an analytic function of $\xi$. Hence, for $\delta=\nu^{2} /\left(8 \Omega \beta_{2}\right)$, we find the alternative definition of the LLL, which, after rescaling by $\beta_{2}$ yields the same LLL as in the isotropic case, that is the space $L$. This definition is equivalent to the one given by Fetter in [13]. Moreover (43) provides (39) with $\kappa^{2}=\gamma \mu_{1} / 2 \beta_{1}$ and since $\gamma \mu_{1} \beta_{1} \sim 2 \varepsilon^{2}, \beta_{2} \sim \sqrt{\left(1-\nu^{2}\right) /\left(1-\nu^{2} / 2\right)}$ and $\gamma \sim\left(4-2 \nu^{2}\right) / \sqrt{1-\nu^{2}}$.

The rest of this section is dedicated to the minimization of $(38)$, which yields two different cases according to the behaviour of $\kappa / \varepsilon^{1 / 3}$.

\subsection{Weakly anisotropic case}

In this section, we assume that

$$
\varepsilon \leq \kappa \ll \varepsilon^{1 / 3} \text {. }
$$

This case is similar to the isotropic case and we derive similar results to the previous section, namely an upper bound given by the Theta function but we lack a good lower bound. The isotropic case is recovered by assuming $\kappa=\varepsilon$.

Theorem 3.1. [7] If (44) is satisfied, and if $\varepsilon \rightarrow 0$, then we have

$$
\frac{2}{3} \sqrt{\frac{2 G \varepsilon \kappa}{\pi}}<I(\varepsilon, \kappa) \leq \frac{2}{3} \sqrt{\frac{2 G b \varepsilon \kappa}{\pi}}+O\left(\sqrt{\varepsilon \kappa}\left(\frac{\kappa^{3}}{\varepsilon}\right)^{1 / 8}\right)
$$

where $b=\gamma(j) \approx 1.1596$ is given by the Abrikosov problem. Moreover, the following function gives the upper bound in (45):

$$
v=\Pi_{L}\left(u_{\tau} \alpha\right)
$$

where $u_{\tau}$ is defined by (29) with $h=1$, and

$$
\alpha(x)^{2}=\frac{2}{\pi \sqrt{b} R_{1} R_{2}}\left(1-\frac{x_{1}^{2}}{\sqrt{b} R_{1}^{2}}-\frac{x_{2}^{2}}{\sqrt{b} R_{2}^{2}}\right)_{+}, \quad R_{1}=\left(\frac{2 G \kappa}{\pi \varepsilon^{3}}\right)^{1 / 4}, R_{2}=\left(\frac{2 G \varepsilon}{\pi \kappa^{3}}\right)^{1 / 4} .
$$


We point out that the lower bound does not include $b$. We expect the energy asymptotics to match the right-hand side of (45). Thus, the lower bound is not optimal. This is confirmed by numerics.

\subsection{Very fast rotation}

In the case where the rotation is fast enough with respect to the anisotropy in the sense that

$$
\kappa \gg \varepsilon^{1 / 3}
$$

we have found a regime unknown by physicists where vortices disappear and the problem can be reduced in fact to a 1D energy.

Theorem 3.2. [7] Assume (47). Then, we have

$$
I(\varepsilon, \kappa)=\frac{\kappa^{2}}{8 \pi}+J \varepsilon^{2 / 3}+o\left(\varepsilon^{2 / 3}\right)
$$

as $\varepsilon \rightarrow 0$, where

$$
J=\inf \left\{\int_{\mathbb{R}} \frac{1}{2} t^{2} p(t)^{2}+\frac{G}{2} \int_{\mathbb{R}} p(t)^{4}, \quad p \in L^{2}(\mathbb{R}) \cap L^{4}(\mathbb{R}), \quad \int_{\mathbb{R}} p^{2}=1\right\}
$$

In addition, if $u$ is a minimizer of $I(\varepsilon, \kappa)$, then

$$
\frac{1}{\varepsilon^{1 / 3}}\left|u\left(\frac{x_{1}}{\varepsilon^{2 / 3}}, x_{2}\right)\right| \longrightarrow 2^{1 / 4} e^{-\pi x_{2}^{2}} p\left(x_{1}\right),
$$

in $L^{2}\left(\mathbb{R}^{2}\right) \cap L^{4}\left(\mathbb{R}^{2}\right)$, where $p$ is the minimizer of $J$.

This is due to the fact that the operator $x_{2}^{2}$ is bounded below by a constant in the LLL. The ground state of the operator $x_{2}^{2}$ is

$$
u\left(x_{1}, x_{2}\right)=\left(\frac{\gamma \beta}{2 \pi}\right)^{1 / 4} \exp \left(-\frac{\gamma \beta}{4} x_{2}^{2}+i \frac{\gamma}{4} x_{1} x_{2}\right)
$$

and every function in the LLL cannot be more localized in the $x_{2}$ direction than (51). This provides an upper bound. The projection onto the space of separate variables in $x_{1}$ and $x_{2}$ is enough to provide the suitable lower bound.

\subsection{Open questions}

The missing information correspond to the intermediate regime where $\varepsilon^{1 / 3} / \kappa$ converges to some limit $\lambda$. We expect that the extension in the $x_{2}$ direction depends on $\lambda$ and wonder whether the condensate has a finite number of vortex lines.

Numerical simulations are still to be performed.

\section{REFERENCES}

[1] Abo-Shaeer JR, Raman C, Vogels JM, Ketterle W, Observation of Vortex Lattices in Bose-Einstein Condensates (2001) Science 292, 476-479.

[2] Aftalion A, Vortices in Bose Einstein condensates, Progress in Nonlinear Differential Equations and their Applications, Vol.67 Birkhäuser Boston, Inc., Boston, MA, (2006).

[3] Aftalion A, Blanc X, Vortex lattices in rotating Bose Einstein condensates, (2006), SIAM J. Math. Anal., Vol 38, p 874.

[4] Aftalion A, Blanc X, Reduced energy functionals for a three dimensional fast rotating Bose Einstein condensates, (2008), Ann. I.H.P., Vol. 25, 339-356.

[5] Aftalion A, Blanc X, Dalibard J, Vortex patterns in a fast rotating Bose-Einstein condensate, (2005) Phys. Rev. A 71023611. 
[6] Aftalion A, Blanc X, Lerner N, Fast rotating condensates in an asymmetric harmonic trap, (2008), condmat-0804.0971.

[7] Aftalion A, Blanc X, Lerner N, (2008), in preparation.

[8] Aftalion A, Blanc X, Nier F, Lowest Landau Level for Bose Einstein condensates and Bargmann transform, (2006) J. Func. Anal. Vol 241, pp 661-702.

[9] Bretin V, Stock S, Seurin Y, Dalibard J, Fast Rotation of a Bose-Einstein Condensate Phys. Rev. Lett. 92, 050403 (2004).

[10] Cohen-Tannoudji CN, in: Les Prix Nobel 1997 (The Nobel Foundation, Stockholm, 1998), pp. 87-108, Reprinted in: Rev. Mod. Phys. 70:707-719.

[11] Cooper NR, Komineas S, Read N,Vortex lattices in the lowest Landau level for confined Bose-Einstein condensates, Phys. Rev. A 70, 033604 (2004).

[12] Cornell EA, Wieman CE, Bose-Einstein condensation in a dilute gas, the first 70 years and some recent experiments, in: Les Prix Nobel 2001 (The Nobel Foundation, Stockholm, 2002), pp. 87-108, Reprinted in: Rev. Mod. Phys. 74, 875-893; Cem. Phys. Cem. 3, 476-493 (2002).

[13] Fetter AL, Lowest-Landau-level description of a Bose-Einstein condensate in a rapidly rotating anisotropic trap, Phys. Rev. A 75, 013620 (2007).

[14] Ho TL, Bose-Einstein Condensates with Large Number of Vortices, Phys. Rev. Lett. 87060403 (2001).

[15] Ketterle W, When atoms behave as waves: Bose-Einstein condensation and the atom laser, in: Les Prix Nobel 2001 (The Nobel Foundation, Stockholm, 2002), pp. 118-154, Reprinted in: Rev. Mod. Phys. 74, 1131-1151 (2002); Chem. Phys. Chem. 3, 736-753 (2002)

[16] Kleiner WH, Roth LM, Autler SH, Bulk Solution of Ginzburg-Landau Equations for Type II Superconductors: Upper Critical Field Region, Phys. Rev. 133, A1226, (1964).

[17] Madison K, Chevy F, Bretin V, Dalibard J, Vortex Formation in a Stirred Bose-Einstein Condensate, (2000) Phys. Rev. Lett., $\mathbf{8 4}, 806$.

[18] Nonnenmacher S, Voros A, Chaotic Eigenfunctions in Phase Space, J. Stat. Phys. 92, 431-518 (1998).

[19] Pethick CJ, Smith H, (2002) Bose Einstein condensation in dilute gases, Cambridge University Press.

[20] Pitaevskii L, Stringari S, (2003) Bose Einstein condensation, International series of monographs on physics, 116, Oxford Science Publications.

[21] Rosenbuch P, Bretin V, Dalibard J, Dynamics of a single vortex line in a Bose-Einstein condensate (2002) Phys. Rev. Lett. 89, 200403.

[22] Stock S, Bretin V, Chevy F, Dalibard J, Shape oscillation of a rotating Bose-Einstein condensate, Europhys.Lett. 65594 (2004).

[23] Watanabe G, Baym G, Pethick CJ, Landau levels and the Thomas-Fermi structure of rapidly rotating Bose-Einstein condensates, Phys. Rev. Lett. 93, 190401 (2004). 